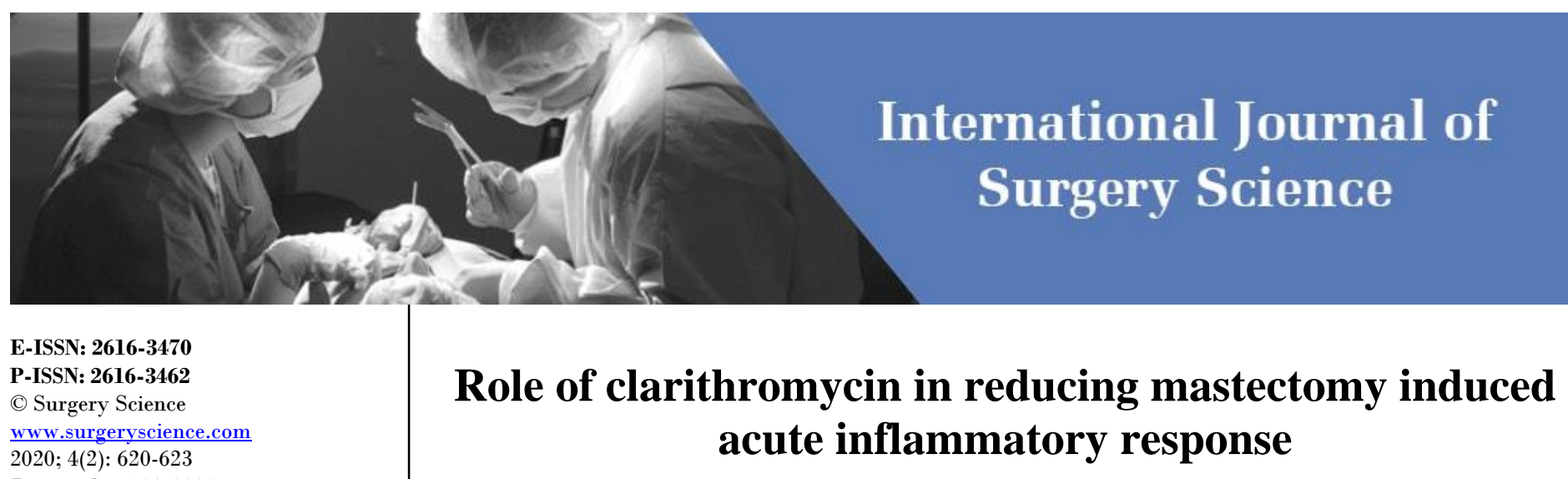

Received: 15-02-2020

Accepted: 16-03-2020

\section{Pankaja SS}

Department of General Surgery, JSS Medical College, JSSAHER, Mysuru, Karnataka, India

\section{Harish Kumar P}

Department of General Surgery, JSS Medical College, JSSAHER, Mysuru, Karnataka, India

\section{Girish Kumar NM}

Department of General Surgery, JSS Medical College, JSSAHER, Mysuru, Karnataka, India
Corresponding Author: Harish Kumar P

Department of General Surgery, JSS Medical College, JSSAHER, Mysuru, Karnataka, India

\section{Pankaja SS, Harish Kumar P and Girish Kumar NM}

DOI: https://doi.org/10.33545/surgery.2020.v4.i2g.454

\section{Abstract}

Background: Surgery induces both inflammation and immunosuppression as part of systemic stress response. Based on the observation that administration of clarithromycin led to an attenuation of the mastectomy induced inflammatory response, we evaluated the anti-inflammatory effects of clarithromycin on mastectomy induced inflammatory response.

Methods: During a 18-month period, 60 patients who underwent modified radical mastectomy (MRM) were randomly allocated to one of the two study groups. In one group patients received oral clarithromycin (500mg) twice daily. Second group was control.

Results: Significant reduction in acute phase reactant, C-Reactive Protein (CRP), Median CRP values were significantly lesser on postoperative day 1 and 2 in the clarithromycin group.

Conclusions: In conclusion, clarithromycin has beneficial anti-inflammatory effect on surgery induced acute inflammatory response.

Keywords: Modified radical mastectomy (MRM), clarithromycin, acute phase reactant, C-reactive protiens (CRP)

\section{Introduction}

Surgery induces both inflammation and immunosuppression as part of systemic stress response. Although generally these effects are minimal and self-limiting, following major surgical procedures, they may become excessive and result in local and systemic inflammatory response syndromes which in turn lead to postoperative morbidity.

Since the time Cuthbertson's and subsequent observations established causal association between trauma and stress response, attempts have been made to attenuate the stress response and improve surgical outcome ${ }^{[1]}$. The various approaches to reduce stress response are use of minimally invasive surgical techniques, adopting regional anesthesia, effective pain relief, minimal use of drains and tubes and use of pharmacological modifiers etc. ${ }^{[2]}$

Anti-inflammatory effects of macrolide antibiotics including Clarithromycin have been assessed in various chronic inflammatory pulmonary conditions. Recently Clarithromycin has been shown to modulate surgery induced inflammatory response effectively, in a guinea pig model ${ }^{[3]}$. Similar effects were seen later in patients undergoing mastectomy. Mastectomy is a suitable procedure for assessing surgery induced inflammatory response and to study the efficacy of novel anti-inflammatory agents as it involves extensive dissection and tissue damage eliciting significant inflammatory response and chance of surgical site contamination is uncommon ${ }^{[4]}$.

As modified radical mastectomy is a routinely done procedure worldwide, we decided to evaluate the potential anti-inflammatory effects of Clarithromycin in these patients.

\section{Methods}

This study conducted in our surgical Department. Patients undergoing modified radical mastectomy for carcinoma breast, were included in this prospective randomized controlled study. Ethical clearance for the study was obtained from the institute ethical committee.

Patients with ulcerated tumor, associated pregnancy, comorbid medical conditions, history of sensitivity to macrolides, already on steroids/antibiotics/immunosuppressants for any reason, who received neoadjuvant-chemotherapy were excluded from the study.

After providing detailed information about the study, written consent was obtained from all patients. Randomization done using odd-even system. 
Patients in the clarithromycin group were started on oral clarithromycin $(500 \mathrm{mg})$, twice daily from the preoperative day and continued till third postoperative day.

Blood samples were collected on the preoperative day to check the baseline level of laboratory parameters such as Total Leukocyte Count, Neutrophils percentage, and C-reactive protein (CRP) levels.

All patients received premedication drugs. All patients were given prophylactic antibiotics as per our institute protocol.

Surgical procedure: Mastectomy was carried out using energy source or scissors, under standard anesthesia protocol (suxamethonium, thiopentone sodium, vecuronium and morphine).
Patients received Intravenous or Intramuscular analgesics in initial $24 \mathrm{hrs}$ and per oral paracetamol till $5^{\text {th }}$ post-operative day for pain management. Blood samples were collected to assess laboratory parameters (TLC, Neutrophils percentage) and CRP levels on $1^{\text {st }}, 2^{\text {nd }}$ and $5^{\text {th }}$ postoperative day (POD).

Statistical analysis: Parameters analyzed using the statistical software package SPSS.10 version.

Mean interval changes were calculated for the laboratory and clinical parameters alone. Mean interval change is the mean of the difference between each POD. The tests employed were Chi square test for all categorical parameters, ANOVA test for all continuous variables, $\mathrm{P}$ value $<0.05$, was considered significant.

\section{Results}

\section{CONSORT diagram showing the flow of participants through each stage of the study}

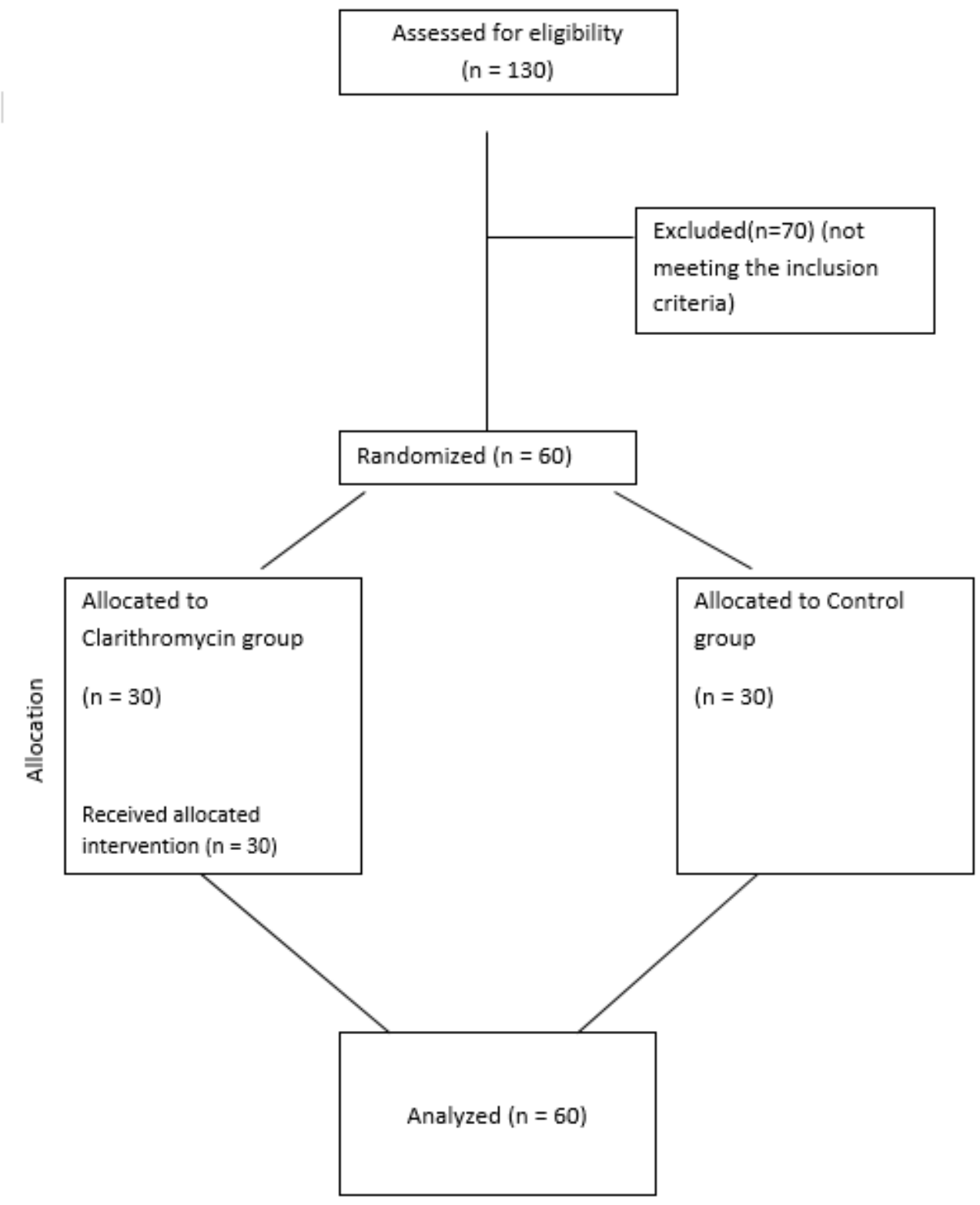

\section{Baseline data}

Mean age at diagnosis and treatment was 46.73 with standard deviation of \pm 10.81 Both study and control groups were comparable in terms of age, weight, body mass index and duration of surgery. All patients were non-smokers as well. (Table 1). 
Table 1: Baseline Data

\begin{tabular}{|c|c|c|c|}
\hline \multirow{2}{*}{} & \multicolumn{3}{|c|}{ Mean \pm SD (Range) } \\
\cline { 2 - 4 } & Control & Clarithromycin & P value \\
\hline Age (in yrs) & $46.73 \pm 10.81(28-70)$ & $44.40 \pm 9.78(22-65)$ & 0.330 \\
\hline Weight (in kgs) & $51.63 \pm 11.35(33-80)$ & $48.87 \pm 9.97(30-72)$ & 0.427 \\
\hline BMI (kg/m $\mathbf{2}^{2}$ & $22.37 \pm 4.53(14.22-30.0)$ & $21.10 \pm 3.99(15.15-32.43)$ & 0.383 \\
\hline Duration of surgery (in mins) & $126.83 \pm 34.43(75-210)$ & $113.67 \pm 27.60(60-180)$ & 0.178 \\
\hline
\end{tabular}

\section{Laboratory parameters}

There was leucocytosis, neutrophilia, on POD1 in both groups and the levels reached preoperative level on POD5.

However in control group, slight neutrophilia and lymphopenia persisted till POD5.

Mean interval changes (MIC) did not differ significantly among the groups, for any of the parameters. (Table $2 \& 3$ )

Table 2: Total Leukocyte count

\begin{tabular}{|c|c|c|c|c|}
\hline \multirow{2}{*}{ Cell type } & \multirow{2}{*}{ Post-operative day(POD) } & \multicolumn{2}{|c|}{ Mean Interval Change \pm SD p-value } \\
\cline { 2 - 5 } & POD 1 & $10483 \pm 3545$ & $10900 \pm 3751$ & 0.66 \\
\hline \multirow{3}{*}{$\mathrm{TLC}\left(\mathrm{Cells} / \mathrm{mm}^{3}\right)$} & POD 2 & $9310 \pm 3032.5$ & $9630 \pm 2372.27$ & 0.65 \\
\cline { 2 - 5 } & POD 5 & $7703 \pm 2694.24$ & $8023.33 \pm 2419.41$ \\
\cline { 2 - 5 } & & & & 0.234 \\
\hline
\end{tabular}

Table 3: Neutrophils percentage

\begin{tabular}{|c|c|c|c|c|}
\hline \multirow{2}{*}{ Cell type } & \multirow{2}{*}{ Post-operative day (POD) } & \multicolumn{2}{|c|}{ Mean Interval Change \pm SD p-value } \\
\cline { 3 - 5 } & 1 & $75.1 \pm 6.53$ & $72.43 \pm 9.20$ \\
\hline Neutrophil (\%) & 2 & $71.96 \pm 7.43$ & 0.20 \\
\hline & 5 & $67.13 \pm 8.58$ & 0.51 \\
\cline { 2 - 5 } & & $0.59 \pm 7.49$ & 0.84 \\
\hline
\end{tabular}

\section{Acute phase reactant:}

C- reactive protein (CRP) levels $\leq 3$ and $>3 \mathrm{mg} / \mathrm{l}$ were considered normal and high respectively. The degree of elevation of CRP was significantly lesser in clarithromycin group on POD1 and POD2 compared to control group ( $\mathrm{p}$ value < 0.001 and 0.078 respectively) (Table 4 ).

Table 4: Acute phase reactant: CRP Comparison of Changes in Acute Phase Reactants

\begin{tabular}{|c|c|c|c|}
\hline \multirow{2}{*}{ Day } & \multicolumn{2}{|c|}{$\begin{array}{c}\text { C-Reactive protein (mg/l) } \\
\text { Median } \pm \text { SD }\end{array}$} & \multicolumn{1}{|c|}{} \\
\cline { 2 - 3 } & Control & Clarithromycin & p value \\
\hline POD1 & $79.66 \pm 39.82$ & $11.12 \pm 21.13$ & $*<0.0001$ \\
\hline POD2 & $81.60 \pm 56.75$ & $56.75 \pm 33.79$ & $* 0.078$ \\
\hline POD5 & $6.45 \pm 12.52$ & $13.8 \pm 12.92$ & 0.762 \\
\hline
\end{tabular}

*statistically significant among the groups

\section{Discussion}

Pharmacological modifiers have been tried during preoperative, intraoperative and postoperative phases to modulate the various components of the stress response induced by surgery and anesthesia. However no single agent is found to be ideal. Currently regional anesthetic techniques utilizing local anesthetics are found to be the most powerful mode available to attenuate the endocrine-metabolic responses (the rise in cortisol, catecholamines, glucagon; hyperglycemia; insulin resistance and negative nitrogen balance) ${ }^{[5]}$.

Pharmacological modifiers have been tried during preoperative, intraoperative and postoperative phases to modulate the various components of the stress response induced by surgery and anesthesia. However no single agent is found to be ideal. Currently regional anesthetic techniques utilizing local anesthetics are found to be the most powerful mode available to attenuate the endocrine-metabolic responses (the rise in cortisol, catecholamines, glucagon; hyperglycemia; insulin resistance and negative nitrogen balance) ${ }^{[5]}$.
Mastectomy provides a good opportunity for the study of immunomodulating effects of drugs like clarithromycin and other Macrolides, In addition to anti-inflammatory effects, clarithromycin has been shown to have significant anti-tumor effects with improved survival in clarithromycin treated non small cell lung cancer patients ${ }^{[6]}$.

In our study clarithromycin showed to decrease the TLC \& Neutrophils but both were found to be statistically NOT significant, however there was a statistically significant decrease in acute phase reactant CRP.

More numbers may be required to show significant difference in TLC and neutrophils, which is an inherent deficiency in this study.

One important limitation of using clarithromycin for immunomodulatory prophylaxis in surgical patients is the possibility of inducing antimicrobial resistance.

Our goal must be to attenuate the deleterious effects of the surgery induced inflammatory response while preserving the ability of the patient to mount an appropriate defense to the stress response of the preoperative period. Modulation of the stress response rather than simple inhibition is likely to confer substantial benefit.

In conclusion, clarithromycin has beneficial anti-inflammatory effect on surgery induced acute inflammatory response. Further placebo-controlled trials with larger numbers of patients and different operative procedures should be conducted to ascertain the beneficial immunomodulatory effects on preoperative stress response.

\section{Acknowledgements \\ Declarations \\ Funding: none}

Conflict of interest: Authors declare no conflict of interest. Ethical approval: Institution ethical clearance obtained. 


\section{References}

1. Wilmore DW. From Cuthbertson to fast-track surgery: 70 years of progress in reducing stress in surgical patients. Ann Surg. 2002; 236(5):643-8.

2. Kehlet H. Multimodal approach to control postoperative pathophysiology and rehabilitation. Br J Anaesth. 1997; 78(5):606-17.

3. Woo PC, Chow LW, Ma ES, Yuen KY. Clarithromycin attenuates the inflammatory response induced by surgical trauma in a guinea pig model. Pharmacol Res. 1999; 39(1):49-54.

4. Chow LW, Yuen KY, Woo PC, Wei WI. Clarithromycin attenuates mastectomy-induced acute inflammatory response. Clin Diagn Lab Immunol. 2000; 7(6):925-31.

5. Yatsunami J, Turuta N, Wakamatsu K, Hara N, Hayashi S. Clarithromycin is a potent inhibitor of tumor-induced angiogenesis. Res Exp Med (Berl). 1997; 197(4):189-97.

6. Wada T, Sata M, Sato J, Tokairin Y, Machiya J, Hirama N et al. Clarithromycin suppresses invasiveness of human lung adenocarcinoma cells. Chemotherapy. 2007; 53(2):7784. 